\title{
Report
}

\section{Das Grundrecht auf informationelle Selbstbestimmung darf weder von inländischen noch von ausländischen Stellen verletzt werden!}

Pressemitteilung der Konferenz der Datenschutzbeauftragten des Bundes und der Länder, 27.06.2013

Die Konferenz der Datenschutzbeauftragten des Bundes und der Länder ist äußerst besorgt angesichts der Enthüllungen über die umfassenden und anlasslosen Überwachungsmaßnahmen des us-amerikanischen und des britischen Geheimdienstes.

Es steht im Raum, dass ein großer Teil des Kommunikationsverhaltens der Menschen in Deutschland ohne ihr Wissen von diesen Geheimdiensten überwacht wird.

Die Konferenz der Datenschutzbeauftragten des Bundes und der Länder erwartet, dass die Bundesregierung alles unternimmt, um die Menschen in Deutschland vor informationellen Zugriffen Dritter zu schützen, die mit der Verfassungsordnung des Grundgesetzes nicht im Einklang stehen. Die Bundesregierung muss für eine restlose Aufklärung des Sachverhaltes sorgen und dabei auch die Frage beantworten, ob deutsche Behörden diese Informationen übermittelt bekamen und verwendeten. Aus Sicht der Datenschutzkonferenz unterstreichen die bekannt gewordenen Überwachungsmaßnahmen die Dringlichkeit, für Europa hohe Datenschutzstandards zu beschließen und sicherzustellen, dass diese auch für staatliche und private Stellen aus Drittstaaten gelten. Die Datenschutzkonferenz appelliert deshalb dringend an die Bundesregierung, sich jetzt in Brüssel für ein hohes Datenschutzniveau und für Regelungen einzusetzen, die umfassende und anlasslose Überwachungsmaßnahmen europäischer wie außereuropäischer Stellen ausschließen.

Die Vorsitzende der Datenschutzkonferenz, die bremische Landesbeauftragten für Datenschutz und Informationsfreiheit, Dr. Imke Sommer, sagte hierzu: „Die Menschen in Deutschland haben ein Recht darauf, dass sich die Bundesregierung aktiv dafür einsetzt, dass das Grundrecht auf informationelle Selbstbestimmung weder von inländischen noch von ausländischen Stellen verletzt wird."

\section{,Berlin Group' fordert besseren Schutz der Privatsphäre beim Web Tracking}

Die Internationale Arbeitsgruppe zum Datenschutz in der Telekommunikation („Berlin Group“), die seit ihrer Gründung 1983 zweimal jährlich unter dem Vorsitz des Berliner Beauftragten für Datenschutz und Informationsfreiheit Dr. Alexander Dix tagt, hat am 15./16. April 2013 in Prag ein Arbeitspapier zu „Web Tracking und Privatsphäre" angenommen. Darin beschreibt die Gruppe gängige Methoden der Sammlung, Analyse und Anwendung von Daten über Nutzeraktivitäten von einem Computer oder anderen Geräten (z. B. Smartphones) aus im Internet, wenn dort verschiedene Diens- te der Informationsgesellschaft genutzt werden. Diese werden zunehmend zu verschiedenen Zwecken zusammengeführt und analysiert; sie reichen von wohltätigen bis hin zu kommerziellen Zwecken der verschiedenen Teilnehmer, die solche Dienste oder Teile davon bereitstellen.

Modernes Web Tracking versetzt Anbieter in die Lage, beinahe jeden einzelnen Aspekt des Nutzerverhaltens im gesamten Internet zu beobachten. Dies birgt das Potenzial für die Erstellung einer vollständigen Übersicht über die Nutzung des Internets einer Einzelperson über potenziell unbegrenzte Zeitspannen - buchstäblich von der Wiege bis zum Grab. Diese Interessen- bzw. Nutzungsprofile können mit vielen Daten aus der "Offline-Welt" angereichert werden und alle möglichen Aspekte des Lebens betreffen, über die Datenmakler Informationen besitzen. Dazu gehören auch Informationen über Finanzen, über Freizeitgestaltung, Gesundheit, politische oder religiöse Überzeugungen sowie Informationen über Aufenthaltsorte.

Diese Entwicklung birgt eine beispiellose Gefahr für die Privatsphäre der Menschen in der Informationsgesellschaft. Sie kann schlimmstenfalls zu einem globalen Panoptikum führen:

Das Pendant in der "analogen Welt" wäre, wenn einem ein Unbekannter ständig über die Schulter schauen würde, ganz gleich, wo man sich befindet (ob auf der Straße oder in der scheinbaren Privatsphäre zuhause), und alles festhielte, was man gerade tut (Fernsehen, online einkaufen, Zeitung lesen und sogar intime Tätigkeiten), ohne dass man wissen kann, wann der Unbekannte gerade zuschaut und wann nicht.

Die Regierungen der Nationalstaaten haben diese besorgniserregende Entwicklung bisher weitgehend der Selbstregulierung durch die Anbieter von Diensten der Informationsgesellschaft überlassen, ohne dass dies zu einer hinreichenden Beachtung der Privatsphäre der Menschen geführt hätte.

In jüngster Zeit hat das World Wide Web-Konsortium eine Arbeitsgruppe eingesetzt, die einen technischen Standard für eine bessere Kontrolle der Analyse des Nutzerverhaltens bei der Nutzung des Internets durch die Betroffenen („Do Not Track“) entwickeln soll. Nach den letzten Entwürfen haben sich dort bisher die Interessen der Industrievertreter durchgesetzt, ohne dass hier der Schutz der Privatsphäre der Menschen hinreichend Eingang gefunden hätte. Es besteht die Gefahr, dass der lobenswerte Ansatz, die Nutzer durch Einführung eines „Do not track“-Schalters in die Lage zu versetzen, die Kontrolle über die Verarbeitung ihrer personenbezogenen Daten zurückzugewinnen, ins Leere läuft.

Die „Berlin Group“ gibt in ihrem Arbeitspapier zur Minimierung der Gefahren für die Privatsphäre der Menschen Empfehlungen an die verschiedenen Interessenvertreter, die im Öko-System des Web Tracking eine Rolle spielen. Sie fordert insbesondere die strikte Beachtung des Grundsatzes der Zweckbindung bei der Verarbeitung personenbezogener Daten sowie eine bessere Information der Nutzer über die Auswirkungen der Verarbeitung ihrer personenbezogenen Daten. Schließlich sollen die Nutzer in die Lage versetzt werden, durch einfache technische Mechanismen eine Kontrolle über die Verwendung ihrer Nutzungsdaten im Internet zurückzu- 